\title{
A monitoring system for detecting repeated packets with applications to computer worms
}

Published online: 8 March 2006

(C) Springer-Verlag 2006

\begin{abstract}
We present a monitoring system which detects repeated packets in network traffic, and has applications including detecting computer worms. It uses Bloom filters with counters. The system analyzes traffic in routers of a network. Our preliminary evaluation of the system involved traffic from our internal lab and a well known historical data set. After appropriate configuration, no false alarms are obtained under these data sets and we expect low false alarm rates are possible in many network environments. We also conduct simulations using real Internet Service Provider topologies with realistic link delays and simulated traffic. These simulations confirm that this approach can detect worms at early stages of propagation. We believe our approach, with minor adaptations, is of independent interest for use in a number of network applications which benefit from detecting repeated packets, beyond detecting worm propagation. These include detecting network anomalies such as dangerous traffic fluctuations, abusive use of certain services, and some distributed denial-of-service attacks.
\end{abstract}

Keywords Computer worms - Anomaly detection . Network security · Intrusion detection · Traffic monitoring

\section{Introduction}

Worms, unlike other malicious code such as viruses and trojans, are capable of self-propagating [60]. The constant threat of worms to the network infrastructure has become one of the major concerns of network equipment designers.

P. C. van Oorschot

School of Computer Science, Carleton University, Canada

E-mail: paulv@scs.carleton.ca

J.-M. Robert

Research and Innovation Centre - Security Group, Alcatel Canada, Canada

E-mail: jean-marc.robert@alcatel.com

M. Vargas Martin $(\varangle)$

University of Ontario Institute of Technology, Ontario, Canada

E-mail: miguel.vargasmartin@uoit.ca
In this paper we propose a router-based monitoring system using Bloom filters with counters (BFWC) [17] to automatically detect repeated packets. This capability is adapted to detect worms which transmit significant numbers of packets within short time periods. The idea is to use BFWCs to count the number of times each packet has been forwarded locally. If the count for one packet exceeds a specified threshold within a given fixed period of time, the BFWC triggers an alarm. (Note that Bloom filters can indeed be implemented in hardware without considerable performance degradation in routers $[13,50]$.) In addition, the detection mechanism provides sufficient information - e.g., identifying a port number or packet signature - to allow some possible reactions. (In contrast, e.g., detecting only that "there is a UDP flood attack in progress" would not suffice to allow an appropriate reaction.) While the monitoring system can also detect abnormal behavior in the network (by identifying the port number responsible for sending significant amounts of repeated packets), in this paper we focus our attention largely on worm detection.

A large class of worms can be characterized by selfpropagating code that selects victims by generating random IP addresses, yielding a propagation pattern giving the impression of a "fan." For example, the Slammer worm [36] arrives at the victim network in one malicious UDP packet; once the infection is completed an infected host sends the malicious packet to as many random IP addresses as possible. Similar behaviour can also be observed in multi-TCPpacket worms such as Code Red [63]. This paper addresses the problem of how to efficiently detect such a propagation behavior locally, and how to locate the monitoring mechanisms in the network infrastructure to use as few monitors as possible.

\subsection{Our contributions}

Our main contributions are: (1) a monitoring system based on stateful analysis of network traffic in routers, using an extension of Bloom filters [4]; (2) a validation of the system 
using limited data sets of network traffic, which suggests that the number of false alarms may be reduced by filtering out a small number of common preidentified nonworm repeated packets, such that they are not processed by the BFWC; (3) addressing the problem of where to locate the monitoring mechanisms in the network infrastructure to use as few monitors as possible, which eases deployment and reduces the impact on the network; this may be of independent interest. We furthermore (4) use simulations to show that deploying BFWCs in routers of an approximate minimum vertex cover may be effective at detecting worms in the early stages of propagation.

While our results, as presented, involve algorithms operating directly on packets - which is the context in which we have carried out this research - in practice our (packet matching) work may be of greater interest if one assumes a device carries out these algorithms after full IP and TCP stream reassembly by independent means (see [15]).

\subsection{Limitations}

As our monitoring system is designed to detect worm packets with identical payloads, it has limitations with respect to worms with varying payloads. BFWCs are not effective against polymorphic worms (i.e., those changing their representation on each new infection, e.g., see Nachenberg [37]), or the simpler subset of encrypted worms (which encrypt the bulk of their payloads using a different key per infection). Even a worm which changes a single byte (e.g., the Witty worm [47], which includes random padding and variable destination port in each packet) can deceive BFWCs. To deal with polymorphic worms that only mutate part of their payload, the monitoring system may use a fingerprinting technique which considers only unchanged portions of the payload such as the Rabin fingerprinting technique [45], which has been previously used in intrusion detection (see $[27,48,49])$. Identifying these unchanged portions is beyond the scope of this paper, and merits attention as an open problem. However, our approach in its present form is of use as a first step, and indeed is capable of detecting "popular" recent worms, almost all of which are nonpolymorphic. (For further discussion of polymorphic worms and "Polygraph" [39], see sect. 2.) While we believe that detecting polymorphic worms is an important problem, in this paper we focus on how BFWCs can be deployed efficiently for detecting nonpolymorphic worms. Also, an attacker could take evasive action through worm instances with packets intentionally fragmented to cause recognition ambiguities [23, 44], thereby escaping our BFWC detection technique. Nonetheless, our results remain interesting in that they may lead towards an effective stream matching algorithm.

\subsection{Outline}

Section 2 briefly overviews related work. In Sect. 3 we present the principles of Bloom filters, leading to the BFWC discussion of Sect. 4. In Sect. 5 we consider the deployment of BFWCs in routers of a vertex cover. Section 6 addresses some important implementation issues. In Sect. 7 we evaluate the performance of the BFWC under selected traffic data sets. Section 8 reports on the performance of the BFWC in a simulated network. Section 9 gives concluding remarks. The Appendix considers the selection of a threshold parameter used.

\section{Related work}

In this section we present a partial summary of strategies against worm propagation. This includes intrusion detection systems (IDS) based on the principle that worms (as well as other kinds of denial-of-service attacks) perform many similar actions within a short period of time. Our approach relies on this same principle.

The CERIAS Group [8] proposes a network anomalybased IDS based on a common propagation technique observed in worms: probing many random IP-addresses in a "short" period of time. It is assumed that legitimate traffic does not exhibit such probing behavior. The idea is to have a sensor constantly monitoring the outbound traffic of a host (or a set of hosts). This sensor triggers an alert if the number of packets of a particular [src_addr, dst_port] pair exceeds a predefined threshold $t$. The counter for each [src_addr, dst port] pair is set to zero every $Q$ units of time. The authors indicate that the implementation of this approach is in progress; details are not provided. A similar approach has been used to detect malware scanning hosts remotely [26].

Williamson [62] pursues an approach based on the same principle. He presents a network anomaly-based intrusion detection and response system against viruses based on the belief that a virus would open many new connections, i.e., connections that have not been seen recently. This behavior is also characteristic of worms. The idea is to maintain a list of $n$ (different) destination IP addresses (the working set) representing the $n$ most recently open connections. Any connection to an IP address not found in the working set is considered a new connection. Before a connection is processed the system checks for newness; if the connection is not new, it is processed normally. Otherwise, the connection is put into a delay queue, whose elements are popped off in FIFO order every $Q$ units of time. An implementation of this approach is presented in [54].

Based on the same principle, Weaver et al. [61] present a worm containment system which throttles connections based on approximate source-destination pair reputation. In this approach, a pair gains/loses good reputation every time a connection attempt between the source and destination succeeds/fails. Based on the same principle, Venkataraman et al. [57] propose a system for detecting superspreaders (i.e., hosts which contact at least a given number of other hosts within a short period of time) at low memory and processing expense. The authors present two basic probabilistic approaches based on hash-based sampling; one of 
them consists of sampling distinct source-destination pairs and counting the number of times each pair is repeated within a short period of time. The other algorithm performs a two-level filtering where the first level consists of filtering out (i.e., not sampling) sources which contact a small number of distinct destinations, whereas in the second level each distinct source-destination pair resulting from the first-level filtering is counted.

Following the same principle, Toth et al. [53] propose a network anomaly-based IDS against worms, involving a firewall on each host, a traffic monitor per local network, and a central analyzer. Each traffic monitor listens to all packets transmitted in that network and collects tuples of the form: [timestamp, src_addr, src_port, dst_addr, dst_port,

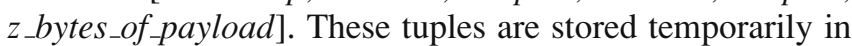
a connection-history table, which is renewed every given fixed period of time. The central analyzer periodically collects lists of tuples from the traffic monitors and infers attacks based on measured values which include the number of times a tuple is repeated, the number of connection attempts to non-existing hosts, and the number of connection attempts to non-existing services. Upon detection of a worm, the central analyzer automatically broadcasts "appropriate changes" to the firewalls' policies to all the hosts in the network. Given the number of issues involved overall (e.g., overhead of reassembling packets, storage capacity required to keep lists of tuples, time to retain the lists, etc.), the impact on network performance is not clear; no performance measurements or implementation is reported.

Based on the same principle, Chen et al. [9] present a worm propagation detection system employing packet matching. An enhanced router maintains two lists of counters with each counter associated with one port. One list is for incoming connections; the other is for outgoing connections. The system matches port counters in both lists and then monitors the number of packets to distinct destination addresses for each of these ports. The system relies on Williamson's observation [62] that users (as opposed to worms propagating to random IP addresses) usually make connections to a small set of addresses. Every predetermined period of time enhanced routers check the number of connections to different addresses. Worm activity is inferred if this number is "significantly" larger than the long-term average. The system is tested with simulations using different router-level topologies. The authors test the system under different scenarios including variations on scanning strategies, network topologies, and parameters of the detection system. No experimentation using real traffic or real network topologies is reported. Unless routers are vulnerable to worm infections, matching incoming and outgoing port counters is not effective in routers which have no LANs attached.

Singh et al. [48, 49] present the "EarlyBird System" for worm detection based on packet payload analysis. Their approach consists of detecting the most popular flows (flows are identified with packet payload hashes), counting the number of source and destination addresses for these flows, and counting the number of connection attempts of these flows to unused portions of IP addresses. The system can detect different malicious packets in real networks. As for almost all systems to date (including that proposed in the present paper), worms employing polymorphism or end-toend encryption evade detection.

Wang et al. [58] propose a network IDS based on $n$ gram analysis (an $n$-gram is a consecutive sequence of $n$ characters or symbols in a text document). The idea is to train the IDS with traffic with no malicious packets into categories according to destination port and size. Then an $n$-gram frequency distribution is computed for each category (they focus on 1-gram analysis). After the training period the IDS compares statistically the $n$-gram frequency distribution of each arriving packet with the $n$-gram frequency distribution of the corresponding category (according to destination port and packet size) using a variant of the well-known Mahalanobis distance. If the result of this comparison is above a threshold the packet is considered malicious. Matrawy et al. [32] propose a network denialof-service mitigation system based on $(p, n)$-gram analysis, where a $(p, n)$-gram is an $n$-gram at byte position $p$. The idea is to categorize traffic according to their $(p, n)$ gram characteristics hoping that disruptive traffic can effectively be separated from non-disruptive traffic. In terms of network overhead, the number of tasks involved in many of these systems [32, 49, 58] raises performance issues warranting further practical validation regarding applicability in core routers. ${ }^{1}$

Newsome et al. [39] present "Polygraph," a system for automatic generation of signatures for polymorphic worms. This system relies on the fact that a number of polymorphic worms need to contain an unchanged portion to be able to exploit the targeted vulnerability (this may not the case for Santy worms, for example, which exploit generic flaws of web applications [29]). The authors present a group of signature generation algorithms which employ the naïve Bayes classifier, and methods for finding signatures by determining the presence of a set of tokens within a sequence of bytes. "Polygraph" relies on appropriate classification of worm and nonworm flows.

Valdes et al. [55] present a visualization technique of network activity. This technique allows visual detection of vertical and horizontal scanning through graphical combinations of source and destination IP addresses and ports. The authors indicate that appropriate entropy analysis may enable this technique for early detection of malicious traffic; see also Onut et al. [41].

Regarding the deployment of sensors in core networks, Park et al. [43] show experimentally that a vertex cover of the Internet can be constructed with approximately $20 \%$ of the total number of nodes. Their results rely on the characteristics of the network underlying the Internet [16]. Park et al. use randomly generated power-law networks to test

1 The EarlyBird system has recently been progressed commercially and advanced (including hardware implementation) by NetSift Inc., which was acquired by Cisco in June 2005. 
the performance of a well-known approximation algorithm for finding a vertex cover. They deploy filtering mechanisms in the vertex cover to detect spoofed addresses. We use the vertex cover results of Park et al. to deploy our monitoring mechanism for detecting repeated packets.

\section{Bloom filters}

In this section we review Bloom filters [4]. A Bloom filter is a hash based method for testing membership of a series of items in a large given set of items, with allowable errors. Bloom filters have been used in a number of different contexts since they were introduced [7, 13, 14, 21, 28, 46, 56]. For example, Snoeren et al. [50] present a traceback system capable of tracing packets delivered by the network in the recent past using Bloom filters. They show that the system is effective (up to a certain number of false positives, inherent to Bloom filters), efficient (requiring storage proportional to $0.5 \%$ of the link capacity), and, perhaps most importantly, they show that it can be efficiently implemented in hardware. Using Bloom filters each router of the network keeps a record of the packets that it sends forward over a certain fixed time window. When a packet needs to be traced back, the system constructs an attack graph indicating the path(s) from which the packet was forwarded along the network. This graph is constructed by checking which routers forwarded this packet.

The idea of Bloom filters is to insert a given set of items into a bit-array, or Bloom-table, as follows. Each item is hashed by $k$ independent hash functions $h_{i}$, for $i=1, \ldots, k$. The resulting value of each of these hash functions is interpreted as an index pointing to an entry of the Bloom-table. Then the corresponding entry of the Bloom-table is set to 1 (initially, each entry is set to 0 ).

To test membership of an item $p$, a similar procedure is followed: $p$ is hashed and if $h_{i}(p)=1$, for $i=1, \ldots, k$, then it is inferred that $p$ is already in the Bloom-table. See Fig. 1.

One way to assess the efficiency of a Bloom filter is by measuring the accuracy of its membership test. If

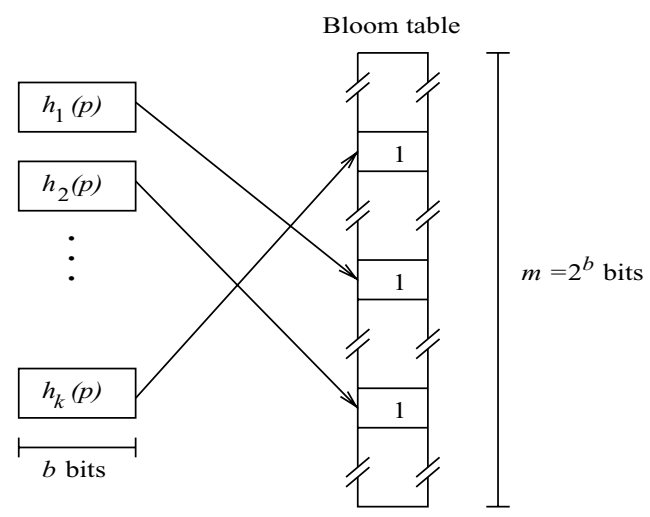

Fig. 1 Illustration of a Bloom filter a membership test is negative, we can be certain that it is correct, i.e., the item $p$ has not been inserted into the Bloom-table. However, if the test is positive, there exists the possibility that the result is incorrect, i.e., arises due to several items $p^{\prime} \neq p$ collectively causing cells $h_{i}(p)$ being set to 1 . We define a false positive as follows.

Definition 1 (false positive) Insert $n$ items $p_{1}, \ldots, p_{n}$ into a Bloom filter. If a membership test for an item $p \neq p_{i}$ (for $i=1, \ldots, n$ ) succeeds, then we call this event a false positive.

Note that the notion of falseness here has to do with the fact that the membership test succeeds as a result of a hashing collision rather than a repeated item.

The number of false positives depends on the number of hash functions $k$, the size of the Bloom-table $m$, and the total number of items to be inserted, $n$. It is desirable to design a Bloom filter which simultaneously minimizes the number of false positives, the size of the Bloom-table, and the number of hash functions. The number of false positives can be approximated as follows. When an item is inserted into the Bloom-table, the probability that any particular entry is set to 1 by one hash function is $1 / m$, and the probability that any particular entry is unchanged is $1-1 / m$. Hence, after inserting $n$ random items using $k$ independent hash functions, the probability that a particular entry is still 0 is $\left(1-\frac{1}{m}\right)^{n k}$. Thus, when we test for membership, the probability $f$ of a false positive equals the probability that the $k$ hash functions all point to entries with value 1 , i.e., $f=\left(1-\left(1-\frac{1}{m}\right)^{n k}\right)^{k}$. For $m$ large, $f$ can be approximated by $f \approx\left(1-e^{-\frac{n k}{m}}\right)^{k}$ which is minimized for

$k=(\ln 2) \frac{m}{n}$.

For $m$ large, we obtain a false positive probability of $f \approx$ $0.6185^{\frac{m}{n}}$. (See e.g., $\left.[17,35].\right)$

There are several studies regarding hardware design of Bloom filters including feasibility studies of incorporating them in network devices without considerable performance degradation (e.g., $[13,50])$.

\section{Bloom filters with counters}

The problem of detecting the "fan" effect produced by worm propagation (see Sect. 1) consists of detecting duplicated packets leaving a network. In particular, we focus on malicious software which employs scanning methods. As reported previously [19], a common scanning strategy consists of sending out many packets with same payload and destination port, but different destination addresses within a short period of time.

Fan et al. [17] introduced BFWC applied to web cache techniques. We use BFWC in a router-based monitoring system. The idea is to enhance the routers of a network (or a subset of them - see Sect. 5) with BFWC to analyze the 
outbound traffic. For each outbound packet $p$, an enhanced router (i.e., a router that implements a BFWC) tracks how many times this packet has been forwarded in the recent past. If the packet has been forwarded more than $t$ times for an appropriate trigger threshold $t$ (precisely the behavior that one would expect from a worm) an alarm is triggered.

To measure the efficiency of a BFWC we need to analyze the expected number of alarms. The value of the threshold $t$ directly affects the expected number of alarms. To determine an appropriate value for $t$ we need to take into account two different factors that may legitimately increase the counters (i.e., in the absence of malicious packets). First, we give some definitions.

Definition 2 (random packet) A random packet is a concatenation of a fixed number $s$ of bits, where each bit may be 0 or 1 with equal probability (0.5). $s$ is the (bit) size of the random packet.

Definition 3 (Class I traffic) Class I traffic is a sequence of $n$ random packets.

Definition 4 (Class II traffic) Class II traffic is a sequence of $n$ packets generated by a legitimate (e.g., non-worm) communication protocol in a network and contains at least one repeated packet.

When we speak of "Class I traffic", we assume that $s$ is sufficiently large relative to $n$ that the probability of any two packets being identical is negligible, or at least relatively small. In real networks, although most packet sequences are not randomly generated, some may look like "Class I traffic" in the sense that no packet is repeated.

We need to consider the increments to the counters caused by "Class I traffic", which increments the counters due to different packets resulting in the same hash value (i.e., statistical hash function collisions). Secondly, we need to consider the increments caused by "Class II traffic", i.e., non-worm traffic that contains repeated packets due to "normal"2 repeated use of particular protocols (e.g., peer-to-peer, Google visits, Yahoo connections). Figure 2 illustrates these two types of traffic. The safety gap is the remaining number of increments allowed or provisioned before the threshold $t$ is reached, i.e., beyond the expected number of Bloomtable entry collisions (increments) contributed by "Class I" and "Class II traffic". This safety gap should be sufficiently large to avoid false alarms due to expected statistical variation in "Class I" and "Class II traffic". "Class I traffic" increments are studied in the Appendix. Increments caused by "Class II traffic" are inherent to the characteristics of the system where the BFWC is deployed and such increments are not a major focus of this paper. However, the results of testing the BFWC under selected data sets (see Sect. 7) partially clarifies how "Class II traffic" affects the counters and what needs to be done to deal with this type of traffic.

\footnotetext{
2 "Normal" will vary depending on the nature of the network and its
} users.

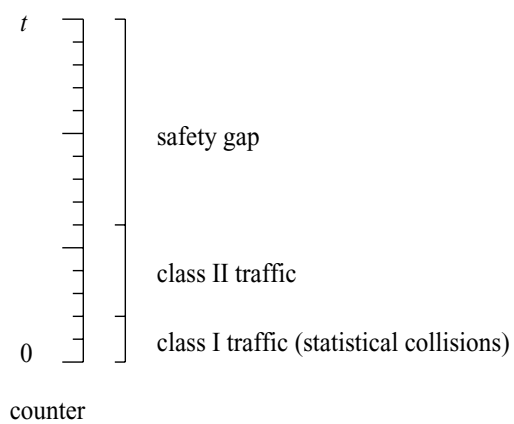

Fig. 2 Types of nonworm traffic that increment a Bloom counter, and the resulting safety gap

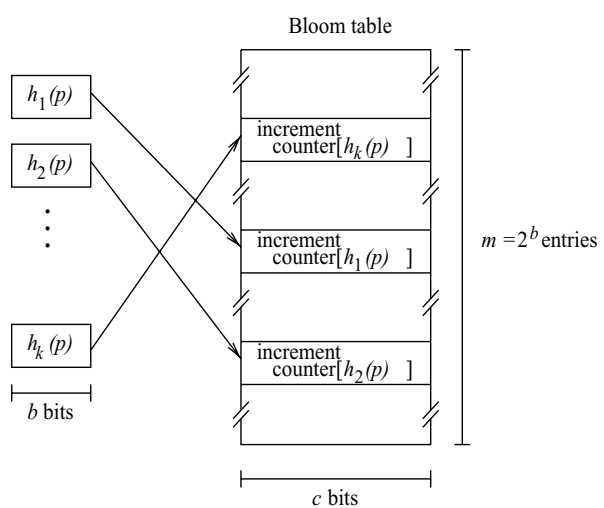

Fig. 3 Bloom filter with counters (BFWC)

Figure 3 illustrates a Bloom filter with counters. Algorithm 4 describes the actions taken for each packet arriving at an enhanced router to be forwarded. Line 2 is a threshold test which triggers if a packet (worm or otherwise) is processed more than $t$ times.

In the Appendix we consider the probability that any $k$ among the $m$ counters have value greater than $t$ each after $n$ packets are processed.

Since memory capacity is limited, the entries of the Bloom-table are reset to 0 every fixed period of time, the reset period. The probability that a counter reaches its maximum value $M$ solely based on "Class I traffic" is low because of the choice of parameters (e.g., size of Bloom-table, number of hash functions, expected number of packets). If a counter reaches its maximum value $M$, increments and wraps around to 0 , important information is lost for the remaining time of the reset period. One way to address this is to use a design whereby the counters remain at the maximum value.

\begin{tabular}{l} 
Algorithm 1 BFWC (packet $p)$ \\
\hline $1 \quad$ for $i \leftarrow 1$ to $k$ \\
$\quad$ counter $\left[h_{i}(p)\right] \leftarrow$ counter $\left[h_{i}(p)\right]+1$ \\
$2 \quad$ if counter $\left[h_{i}(p)\right]>t$ for all $i=1, \ldots, k$ then \\
trigger alarm \\
$3 \quad$ else process $p$ as usual
\end{tabular}




\section{Enhanced routers}

Consider BFWCs (as described in Sect. 4) with "appropriate" values for $k$ and $m$ according to the expected traffic volume $n$, and a threshold $t$ with a reset period $r$. Suppose that these BFWCs are deployed in each router of the network. Worms spreading at $t$ or more worm packets per second from a host would be detected by the first router that forward these packets. In this way, enhancing all the routers of a network would facilitate detection. However, costs and complexity of deployment might make it impossible or impractical to enhance every single router of a network. Therefore, we are interested in minimizing the number of enhanced routers while still being able to detect worms efficiently. In this section we describe how we can take advantage of Internet connectivity properties to find an appropriate small set of routers to enhance.

Consider a graph representing a network of autonomous systems where each node represents an autonomous system. Internet topology follows power-law relationships [16], including: the number of nodes with d neighbors is proportional to $d^{-c}$, where $c>1$.

Several experimental studies have attempted to find an accurate value for $c$ (e.g., [6, 16]). Since the power-law property induces hubs in the network (i.e., routers attached to "many" other routers - see [59]), we suggest that enhancing the routers of a small vertex cover including the hubs would be advantageous. A vertex cover of an undirected graph $G=(V, E)$ is a set of vertices $C \subseteq V$ such that for each link $(u, v) \in E, u$ or $v$ or both are in $C$. Given $G$ and a positive integer $z \leq|V|$, the vertex cover problem is to find a vertex cover $C$ such that $|C| \leq z$, if one exists. This problem is NP-complete [20].

Park et al. [43] show experimentally that a vertex cover of the autonomous-system-level Internet can be constructed with approximately $20 \%$ of the total number of nodes, and use random power-law networks to test the performance of a standard greedy algorithm [10, Sect. 35.1] - which we call greedy $V C$ - for finding an approximated minimum vertex cover. This algorithm iteratively selects a node of highest degree and adds the node to the vertex cover, deleting the associated edges until all links are covered. We expect that enhancing the routers as selected by algorithm greedy $V C$, will be efficient in detecting a worm since the routers of the vertex cover tend to be the ones with most neighbors, and therefore the ones that will likely forward most of the traffic in the network (see results of Sect. 8).

\section{Implementation issues}

In this section we discuss a few important implementation issues regarding BFWCs, four in particular: the packetsubset that is input to the hash functions, the nature of the hash functions themselves, the amount of memory required to store the Bloom-table, and the length of the reset period. While additional issues arise in an actual implementation, these are the most fundamental ones.
To be able to detect the "fan" produced by worm propagation we must look only at those portions of the packet that do not change. Our selection criteria for extracting a packet-subset is to retain those portions of the packet which we expect to be identical in a propagating worm (see Sect. 9 for comments regarding polymorphic worms). For example the destination IP address would vary, therefore this field should be excluded. For most worms, the destination port however, is expected to be constant. At the same time we want the packet-subset to contain enough information to avoid having non-identical packets which have the same packet-subset. Thus, we propose assembling packet-subsets of the form: [dst port, payload]. Taking packet-subsets of this form prevents worms from evading detection by simply spoofing (varying) source IP addresses which would result in distinct hashes; i.e., worm packets not being counted by the same $k$ counters of the Bloom-table. In addition, this packetsubset allows the detection of worm packets coming from different IP sources. However, by omitting the source IP address in the packet-subset, flashcrowds (i.e., sudden transmission of a significant number of packets from different IP addresses) of legal packets yielding equal packet-subsets may trigger false alarms; e.g., the same http request made at the same time by a considerable number of users in a corporation network, or any broadcasting application as online gaming. The destination port helps us to identify the vulnerable application.

It is important to use appropriate hash functions according to the requirements on performance and security. Several papers have discussed performance measures of hash functions implemented in both hardware and software. For example Grembowski et al. [22] show experimentally that SHA-512 implemented in hardware performs surprisingly well (i.e., with not much additional performance degradation) in terms of speed compared to other widely-known cryptographic hash functions. Nevelsteen et al. [38] compare several MAC algorithms against universal hash functions implemented in software and find that universal hash functions perform better than MAC algorithms in general. However, for our present purposes, we expect that even simple linear hash functions may suffice and provide performance advantages (e.g., see [24, p. 151]). For the purposes of our present research also, we are less concerned about advanced attacks such as those proposed by Crosby et al. [11]; depending on his objectives, a worm writer might achieve greater success by spending additional time crafting better worms than by trying to exploit details of hash functions employed in a detection mechanism deployed in some subset of a large network. An attacker, however, could carefully fabricate packets that provoke false alarms due to hash collisions (i.e., different packets resulting in the same hash value, as described by Crosby et al.). This attack represents a potential problem requiring further consideration, and argues against the use of linear hash functions.

As discussed in Sect. 3, the efficiency of a BFWC depends on the total number of packets $n$ expected to be processed over a fixed reset period $r$, the number of hash 
functions $k$, the number of counters $m$ in the Bloom-table, and the bit size of each counter. The objective is to achieve an acceptable level of false alarms with reasonable memory size. Several factors need to be considered to design realistic and efficient Bloom filters, as illustrated in the following paragraphs.

Suppose we have a relatively low bandwidth OC-3 router interface $^{3}$ and 520 KBytes of memory available for the Bloom-table. ${ }^{4}$ First, if the threshold $t$ can fit within 4 bits (see Appendix) then we can allocate two counters per byte, giving a Bloom-table with $m=1,040,000$ counters. Secondly, considering an average packet size of 1000 bits, this router can forward at most about 150,000 packets per $\mathrm{s}$. Now, suppose we want to detect worm propagation within 1$\mathrm{S}$ windows, then a reset period of $r=1 \mathrm{~s}$ yields $n=150,000$ packets. Thirdly, the number of hash functions $k$ can be obtained from Eq. (1): $k=\ln (2) \cdot 1,040,000 / 150,000=4.8$ (to reduce overhead we can choose $k=4)$. Finally, an appropriate trigger threshold $t$ under "Class I traffic" can be selected as explained in the Appendix. With these parameters and $t=15$ the probability of a false alarm after one second of "Class I traffic" is approximately $1.52 \times 10^{-64}$ (see Appendix), or $4.79 \times 10^{-57}$ in 1 year $\left(3.15 \times 10^{7} \mathrm{~s}\right)$, leaving ample room to accommodate links of sufficiently higher bandwidth.

Similarly, for an OC-192 router interface (with a capacity of $n=10,000,000$ packets per second, considering an average packet size of 1000 bits) with $4 \mathrm{MB}$ of available memory, a BFWC with $m=8,000,000$ counters (i.e., two counters per byte), $k=4$ hash functions, and $t=15$, would have a false alarm probability of approximately $2.27 \times 10^{-17}$ under one second of "Class I traffic," or $7.15 \times 10^{-9}$ over 1 year. Using more memory, for example $64 \mathrm{MB}$ (vs. $4 \mathrm{MB}$ ), would decrease this rate dramatically.

\section{Performance of BFWCs under specific traffic samples}

In our evaluation we use packet subsets of the form: [dst_port, payload], as discussed in Sect. 6. It is clear that the BFWC would detect packets with identical packet-subsets forwarded at more than $t$ packets per reset period. However, we expect that the number of non-worm packets with identical packet-subsets is not sufficiently large to trigger false alarms.

In our preliminary analysis, we use three different data sets to test a BFWC with the parameters described in Table 7: one hour of incoming traffic to our internal lab (outside), one day of traffic generated within our internal lab (inside), and 1 day of traffic of the well known DARPA data set [34] (the last two are known to contain no worm packets). The data sets consist of $113,463,37,379$, and 1,476,391

\footnotetext{
3 OC stands for optical carrier. One OC-3 link has a capacity of 155.52 Mbps.

4 An OC-3 router interface is not realistic compared to core routers which may have several OC-192 interfaces, however one OC-3 router interface can be well used as an illustrative example.
}

Table 1 Most frequent destination ports of the outside traffic

\begin{tabular}{rrrrrr}
\hline Port & Freq. & Port & Freq. & Port & Freq. \\
\hline 80 & 43,582 & 137 & 382 & 513 & 40 \\
32,777 & 38,815 & 138 & 346 & 1,715 & 27 \\
1,985 & 7,771 & 21 & 291 & 1,667 & 26 \\
16,080 & 6,455 & 34,852 & 124 & 1,699 & 26 \\
32,771 & 3,111 & 32,781 & 113 & 1,674 & 25 \\
32,787 & 3,060 & 53 & 108 & 1,683 & 25 \\
32,788 & 2,713 & 22 & 106 & 1,706 & 25 \\
520 & 2,429 & 427 & 71 & 1,689 & 24 \\
631 & 2,324 & 3,127 & 47 & 32,772 & 22 \\
135 & 770 & 445 & 47 & 1,096 & 21 \\
\hline
\end{tabular}

Table 2 Most frequent destination ports of the inside traffic

\begin{tabular}{rrrrrr}
\hline Port & Freq. & Port & Freq. & Port & Freq. \\
\hline 53 & 7,830 & 34,295 & 552 & 33,298 & 270 \\
631 & 6,118 & 32,886 & 420 & 3,932 & 263 \\
9,100 & 5,489 & 33,175 & 414 & 68 & 192 \\
32,795 & 2,575 & 33,788 & 414 & 67 & 188 \\
138 & 2,088 & 1,028 & 319 & 3,179 & 177 \\
123 & 1,544 & 33,300 & 315 & 40,216 & 156 \\
38,729 & 1,455 & 38,833 & 300 & 3,213 & 126 \\
137 & 1,198 & 39,135 & 287 & 1,298 & 126 \\
32,775 & 615 & 32,770 & 285 & 3,159 & 114 \\
32,782 & 600 & 50,566 & 272 & 34,296 & 112 \\
\hline
\end{tabular}

Table 3 Most frequent destination ports of DARPA data set

\begin{tabular}{rrrrrr}
\hline Port & Freq. & Port & Freq. & Port & Freq. \\
\hline 23 & 398,734 & 123 & 10,511 & 15,901 & 6,395 \\
80 & 202,921 & 21,262 & 8,983 & 16,510 & 6,143 \\
22 & 85,028 & 24,638 & 8,580 & 18,486 & 6,087 \\
25 & 40,639 & 550 & 8,124 & 30,865 & 5,563 \\
53 & 28,354 & 17,258 & 7,963 & 20,551 & 5,334 \\
161 & 19,992 & 23,308 & 7,536 & 20 & 5,156 \\
32,770 & 19,413 & 16,933 & 6,856 & 14,942 & 5,024 \\
15,574 & 14,177 & 16,586 & 6,757 & 15,037 & 4,798 \\
12,862 & 10,891 & 29,810 & 6,626 & 4,702 & 4,356 \\
1,132 & 10,884 & 21 & 6,554 & 17,782 & 4,349 \\
\hline
\end{tabular}

TCP and UDP packets, with average packet size of 10,464, 7769 and 3011 bits per packet (respectively). The destination port frequency of the data sets are presented in Tables 1, 2, and 3. The predominant destination ports in the outside traffic (ports 80 and 32,777) correspond to http (web) and rpc (remote service requests) packets, whereas the most frequent ones in the inside traffic $(53,631$, and 9100) correspond to DNS (domain names address resolution) and printing protocols. As for the DARPA data set, the traffic is predominantly composed of telnet port 23 (remote login) and http packets on port 80. It is important to note that the incoming traffic to our internal lab had been pre-screened by one or more university firewalls, and thus a number of malicious packets may not have reached our lab. Although our data sets fall well short of the traffic volume in core routers - and further evaluation with representative traffic is necessary for higher confidence-we believe they validate the overall design 
principles of our approach. Similarly, despite known flaws in the DARPA data set [33], it suffices for a preliminary analysis.

To avoid false alarms due to repeated nonworm packets we need to adjust the BFWC according to the predominant (nonworm) repeated traffic in the data sets. For our lab data set, we inserted all the outside 113,463 and the inside 37,379 packets into the BFWC. For the outside traffic we found that packets with the following destination ports triggered false alarms: 1985 (Hot Standby Router Protocol), 520 (local routing process), and 631 (Internet Printing Protocol). We filtered out packets with these destination ports, such that they are not processed by the BFWC. ${ }^{5}$ After this filtering, the BFWC triggered no false alarms (on these data sets) ${ }^{6}$ and moreover was able to detect Slammer packets [36] trying to infect new targets. We were not expecting these latter packets in our network, and thus were pleasantly surprised (by their detection, rather than their presence; the absence of other worms generally present in today's Internet traffic likely resulted from enterprise-level filtering of worm traffic prior to this traffic reaching our outside lab link, as noted earlier). The detection of incoming Slammer packets confirms the effectiveness of BFWCs since incoming worm packets are more difficult to detect, as they may arrive at slower rates than if they were generated from within our lab.

For the inside traffic, no packets had to be filtered out. The BFWC did not trigger false alarms under this traffic; this was as expected, in traffic with no worms.

As for the DARPA data set, we filtered out several destination ports: 520, 161 (Simple Network Management Protocol), 6667 (Internet Relay Chat), and 1270 (OPSMAN). We inserted sequences of 155,550 packets each (the maximum number of packets per second that can be forwarded by a router with 3 OC-3 interfaces, considering an average packet length of 3000 bits per packet). After filtering packets with these destination ports, the BFWC triggered no false alarms, which is what we expected since the data set is known to have no malicious packets.

While obviously additional testing is required on larger data sets representing true Internet traffic, our preliminary results show the effectiveness of our system and suggest that the approach may indeed be effective in core routers.

\section{Simulations}

BFWCs have to be tuned up to take into account environment variables and characteristics inherent to the network, such as type of traffic (e.g., predominantly peer-topeer), network topology fluctuations (e.g., constant failures or changes will trigger routing control packets), network

\footnotetext{
5 These adjustments may vary for different networks depending on factors such as configuration settings, predominant applications, network disruptions, etc.

6 We do not claim zero false alarms for arbitrary Internet traffic, e.g., as might be seen by a backbone router. Again, testing on additional data sets would greatly improve our evaluation, although no data set is representative of all possible classes of real world traffic.
}

Table 4 Characteristics of networks used in simulation

\begin{tabular}{llrr}
\hline & ISP 1 & ISP 2 & ISP 3 \\
\hline Minimum link delay & $1 \mathrm{~ms}$ & 1 & 1 \\
Maximum link delay & $17 \mathrm{~ms}$ & 29 & 44 \\
Number of routers & 108 & 87 & 79 \\
Number of links & 153 & 161 & 147 \\
Routers in vertex cover & 33 & 46 & 37 \\
\hline
\end{tabular}

speed and volume of traffic, available memory in routers, etc. The parameters of the BFWC used in the simulations are determined as discussed in Sect. 6. We note that while our motivation here was for packet matching, the simulation results appear to apply equally for stream matching, although more tuning may be required to address repeated context in streams. (We thank an anonymous referee for this observation.)

The goals of the simulations are (1) to determine an approximation of how efficient the BFWC is in detecting a worm when the BFWC is installed in the routers of an approximate minimum vertex cover and when all the routers of the network are enhanced, and (2) to determine an approximation of how many infections of a particular worm have been perpetrated by the time the worm is first detected by one BFWC.

Several network and worm simulators were considered [30, 40, 42, 52]. In our simulations we use NS-2 [40]. NS-2 provides flexibility on the topology of the network, making it possible to define "large" networks with a reasonable level of detail. The simulations were performed over the backbone topologies of three real ISPs. These topologies were made available by the Rocketfuel project $[1,31,51]$, an Internet measurement tool. The simulation also uses real link delays, also made available by the Rocketfuel project. The characteristics of the ISP topologies used in our simulations are summarized in Table 4.

In our simulations, each node represents a router connected to a local area network (LAN). Internally, the simulator treats each router as a collection of hosts (e.g., clients, servers) on a LAN, as illustrated in Fig. 4. To simplify the simulations, we assume that each router has a LAN associated with it. ${ }^{7}$ Each router has a disjoint set of IP addresses associated, which represent the hosts of the LAN accessible through this router. To simulate IP addresses that do not reside within this ISP (i.e., foreign hosts), we assume that these addresses are reachable through arbitrary routers (assigned at the beginning of the simulation) of this ISP (these foreign hosts can also be thought of as nonvulnerable hosts residing within this ISP).

We simulated a UDP worm that propagates in a single 400-byte UDP packet (not including the required headers) to a non-predetermined number of random IP addresses. The operation of this worm is described in Algorithm 2.

\footnotetext{
7 In simulations, routers themselves are not considered to be hosts.
} 


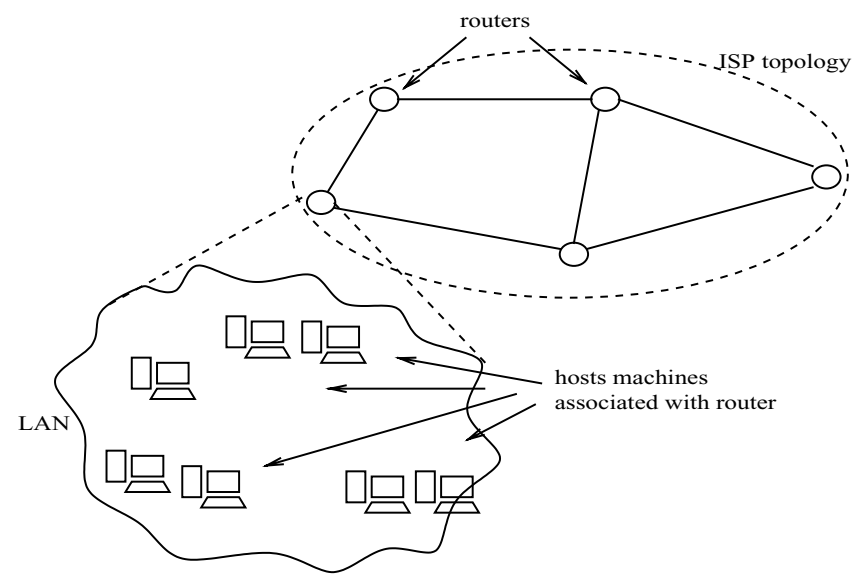

Fig. 4 Example of network topology used in simulations

\section{Algorithm 2 Simulated_UDP_Worm()}

Upon reception of a worm packet, repeat forever:

1 Send copy of the worm packet to a random IP address (i.e., host) using a pseudo-random number generator.

2 Wait for $D$ seconds (the delay $D$ determines the stealthiness of the worm; a larger delay makes detection more difficult).

The propagation of a worm can be modeled with the "generic epidemic model" [12]. This model divides the hosts into two disjoint sets: susceptible and infectious. Infected hosts remain infected for an undetermined period of time (i.e., hosts do not transition from infectious to susceptible). Assuming that the network topology does not change (i.e., there are no new hosts or links, nor failures), and that there are no delays in the transmission lines, the number of infectious hosts at time $T, i(T)$ can be modeled with the following equation:

$\frac{\mathrm{d} i(T)}{\mathrm{d} T}=\beta \cdot i(T) \cdot s(T)$, where $s(T)$ is the number of susceptible hosts at time $T$ and $\beta$ is the propagation rate of the worm (i.e., the number of victims a single infectious host attempts to infect per unit of time).

Initially, in our simulations, all the hosts of the network are susceptible, having a process listening at port 0 , which is assumed to be the service being exploited by the worm. The propagation rate, $\beta$, depends primarily on processor power and bandwidth available. We assume that hosts from other ISPs do not try to infect hosts of the ISP in question; in other words, we assume other ISPs are effectively preventing the spread of the infection.

To simulate multiple infected hosts within a router, whenever a host becomes infected, the propagation rate of worm packets leaving this router is increased accordingly to reflect the number of infected hosts within the LAN attached to this router. If a host attempts to infect another host residing within the same LAN, the attached router will not be able to see the worm packet, and consequently, even if this router was enhanced, the BFWC would not be able to count the worm packet. Thus, we do not detect worm packets propagating within a LAN.

Constant-rate UDP packets representing nonworm traffic are transmitted between random pairs of hosts. The rate of UDP packets transmitted by a host is fixed to 200 packets per s. The payload of each packet is a string of 500 random bytes (see Definition 3). This traffic was generated using the NS-2 built-in generator.

The traffic generated by the underlying routing mechanism should also be considered as part of nonworm traffic. The NS-2 simulator comes with several built-in routing algorithms, including distance vector algorithms [3]. Our simulation was configured to use the built-in implementation of the Distributed Bellman-Ford routing algorithm [3]. Route information packets are thus inherently added to the set of nonworm packets.

The simulation lets us easily adjust the parameters of the network, the BFWC and the simulator itself. The configuration of the three ISP networks used in the simulations is shown in Table 5. The parameters of the worm and nonworm network traffic are listed in Table 6. The nonworm

Table 5 Network parameters used in the simulations

\begin{tabular}{|c|c|}
\hline Parameter & Value or configuration \\
\hline Address & $\sim 3 \times 2^{16}(\mathrm{a}$ \\
\hline Number of hosts associated with each router (s & 100 (empirically set; Rocketfuel does not provide a value for thi \\
\hline Which routers to "enhance" & Routers as selected by an approximate minimum vertex cover \\
\hline
\end{tabular}

Table 6 Worm and nonworm traffic used in the simulations

\begin{tabular}{ll}
\hline Parameter & Value \\
\hline Worm payload & Fixed sequence of 400 bytes within one UDP packet \\
Infection attempts per infected host & 1 per worm delay period, $D$ \\
Worm delay, $D$ & $0.5 \mathrm{~s}$ \\
Nonworm payload & Random sequence of 500 bytes within a single UDP packet \\
Rate of nonworm packets & 200 packets/second, each 500 bytes \\
\hline
\end{tabular}


Table 7 BFWC parameters used in the simulations

\begin{tabular}{ll}
\hline Parameter (see Fig. 3) & Value \\
\hline Size of hash values, $b$ & 20 bits $\left(b=\log _{2} m\right)$ \\
Entries in Bloom-table, $m$ & $1,048,576\left(m=2^{b}\right)$ \\
Bits per counter, $c$ & 4 \\
Hash functions, $k$ & 4 \\
Threshold $t$ & 15 \\
Reset period $r$ & $1 \mathrm{~s}$ \\
\hline
\end{tabular}

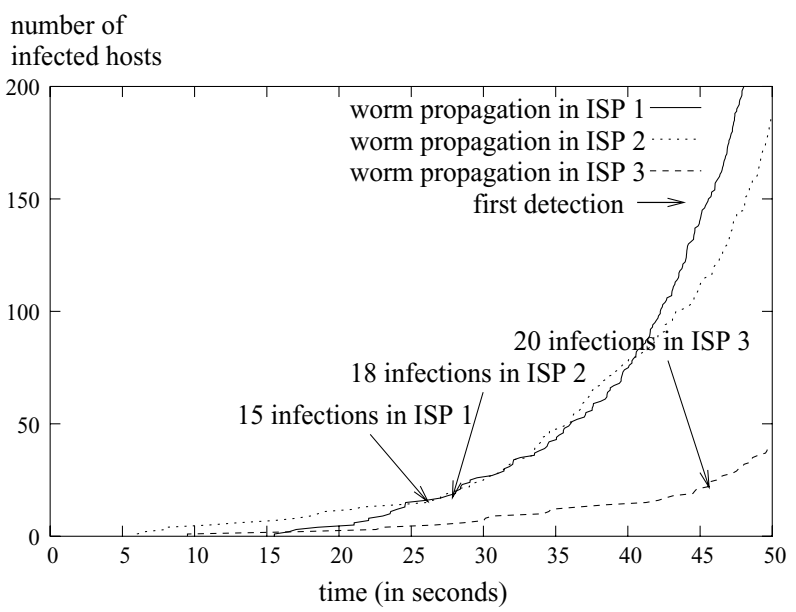

Fig. 5 Detection of the worm enhancing the routers of an approximate minimum vertex cover

traffic can be classified as "Class I traffic" (see Sect. 4). The parameters of the BFWC were set up as discussed in Sect. 6, assuming that the routers have about $520 \mathrm{KBytes}$ of memory available for the Bloom-table and that their maximum throughput is around 150,000 packets per second; a network operator should be able to adjust the BFWC parameters according to the characteristics of the underlying network and its predominant traffic (see Sect. 6). The parameters of the BFWC appear in Table 7.

The simulations were performed over three real ISP topologies with real link delays (see Table 4). The infection is started by a random host within the network. Figure 5 shows how the BFWC is able to detect the worm at its early stage of propagation. The curves show the number of infected hosts (cf. Fig. 4) over time. The arrows show the first detection of the worm by an enhanced router.

The simulations results suggest that enhancing the routers of an approximate minimum vertex cover with BFWCs may be effective in detecting a worm within a reasonably short period of time, e.g., before the worm spreads to $1 \%$ (e.g., per Fig. 5, 20 infections out of $79 \times 100$ hosts for ISP 3 ) of the (vulnerable) hosts in the entire network.

Also with additional experimentation we found that, with the parameters of our particular simulations, enhancing all the routers of the network is essentially no more effective than enhancing only those in an approximate minimum vertex cover. This fact becomes clear when we analyze the scenario. For example, Fig. 6 depicts a portion of the (nongeographic) topology of ISP 1. According to algorithm greedyVC, the routers with most neighbors are most likely to be enhanced, and these routers are the ones forwarding most traffic in the network. For instance, assume that router 93 (top right) in the figure has reset period $r=1 \mathrm{~s}$ and threshold $t=15$. Then it is unable to detect a worm propagating from within its associated LAN at less than 15 packets per second because its Bloom-table is reset every second. The alarms are triggered due to high volumes of worm packets collectively sent by a significant number of hosts. Therefore, even if all the routers were enhanced, a worm is most likely first detected by one of the routers with most neighbors, which most probably belongs to the vertex cover.

\section{Concluding remarks}

We have presented a router-based monitoring system using Bloom filters with counters (BFWC) for detecting worms in the propagation phase. It is based on stateful analysis of network traffic in the routers of a network of LANs, and the principle that worms send out many similar packets within a short period of time. A variation of Bloom filters is used to count the number of times that a packet is repeated. Our simulation results suggest that this approach may be effective in detecting a worm at the early stages of propagation. Further testing under real network conditions and additional data sets is necessary for a rigorous evaluation to answer critical questions about the utility and efficiency of the approach. As noted in Sect. 1, BFWCs are not effective against polymorphic or variable-key encrypted worms.

Our system cannot distinguish between repeated worm packets and repeated nonworm packets. Though this situation must be appropriately addressed (see next paragraph), this "feature" allows our approach to have applications beyond worm detection such as detection of dangerous traffic fluctuations, abusive use of certain services, and distributed denial-of-service attacks. This characteristic of our system does not prevent it from being useful for worm detection since we expect the frequency of repeated worm packets to dramatically exceed that of repeated nonworm packets (see safety gap in Fig. 2), except for "stealth" worms.

An additional solution to preventing repeated nonworm packets from triggering false alarms is to preidentify such packets where possible, and decrement corresponding Bloom-table counters every fixed period of time. This might be viewed as part of the network-specific "tuning".

Further evaluation should include testing the BFWC technique on data sets with significant numbers of packets of particular legitimate protocols (e.g., peer-to-peer) and other legitimate traffic including, e.g., flashcrowd or slashdot-effect behaviour at core router rates - which we expect to be susceptible to false alarms; and determining the tuning required under such traffic. Other follow-on work may involve: exploring how inclusion of more fields in the packet-subset affects the rate of undetected worms; running simulations under different worm propagation rates, 


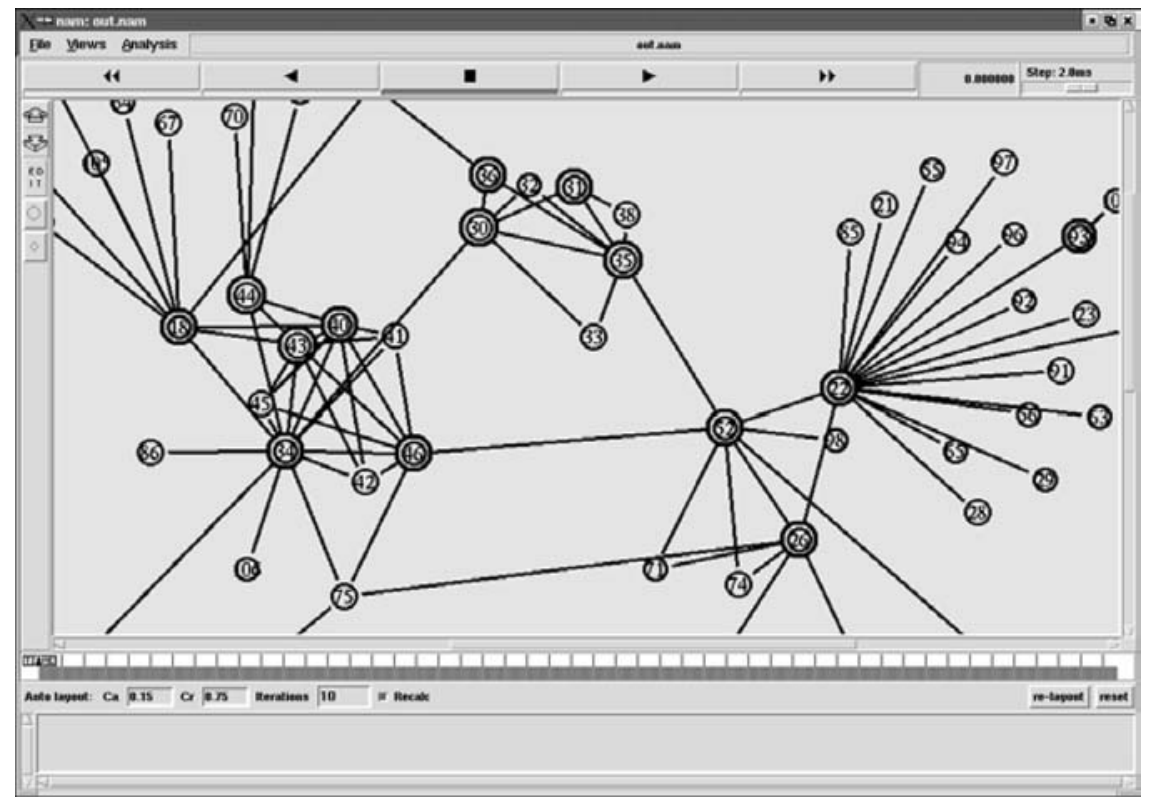

Fig. 6 Partial topology of ISP 1. Each node represents a router. Enhanced routers are illustrated with double circle

and with fewer enhanced routines than those of a vertex cover; and enhancing only routers with particular characteristics (e.g., capacity, type of predominant traffic, number of neighboring routers, etc.).

Acknowledgements Some major parts of this research were carried out while the third author was a post-doctoral student in the School of Computer Science, Carleton University. We thank Javier Govea and Tomás Pospíchal for their valuable help on the simulations and the Poisson approximation to the distribution of counters' values of the Bloom-table (respectively), and anonymous reviewers for their comments which considerably improved the presentation and positioning of this paper. We acknowledge the support of NCIT, MITACS, and Alcatel Canada. The first author is Canada Research Chair in Network and Software Security and acknowledges the support of an NSERC Discovery Grant, and the Canada Research Chairs Program. The third author acknowledges the support of an NSERC Discovery Grant.

\section{Appendix: Determining the repeated packet threshold in $\mathrm{BFWCS}$}

In Sect. 4 we describe Bloom filters with counters (BFWC). Here, we describe a method for generating a table to allow the appropriate selection of a threshold $t$ of repeated packets - at which an alarm is triggered - under "Class I traffic". We first review some basic statistical concepts.

Definition 5 (Bernoulli trials) Bernoulli trials are repeated independent trials with only two possible outcomes for each trial and their probabilities remain the same over time: outcome success has probability $p$, and outcome failure has probability $q=1-p$.

Often one is interested in the probability of obtaining $s$ successes out of $n$ Bernoulli trials regardless of the order of occurrence. This probability has a Binomial distribution.

Theorem 1 [18] Let $b(s ; n, p)$ be the probability that $n$ Bernoulli trials with probabilities $p$ for success and $q=1-p$ for failure result in $s$ successes and $n-s$ failures $(0 \leq s \leq n)$. Then

$b(s ; n, p)=\left(\begin{array}{l}n \\ s\end{array}\right) p^{s} q^{n-s}$.

For large $n$ and small $p$, a direct evaluation of $b(s ; n, p)$ may be impractical. In this case, we can use the Poisson approximation to $b(s ; n, p)$ :

$b(s ; n, p) \approx p(s ; \lambda)=e^{-\lambda}\left(\frac{\lambda^{s}}{s !}\right)$

where $\lambda=n p$.

A generalization of the Binomial distribution for $m$ possible outcomes for each trial is the multinomial distribution [18]. The possible outcomes for each trial are denoted $E_{i}($ for $i=1, \ldots, m)$ and the probability of each outcome is denoted $p_{i}$ (for $i=1, \ldots, m, \sum_{i=1}^{m} p_{i}=1$, and $p_{i} \geq 0$ ). In a multinomial distribution, the probability that in $n$ trials $E_{1}$ occurs $s_{1}$ times, $E_{2}$ occurs $s_{2}$ times, etc., is:

$\frac{n !}{s_{1} ! s_{2} ! \cdots s_{m} !} p_{1}^{s_{1}} p_{2}^{s_{2}} \cdots p_{m}^{s_{m}}$

where $s_{i}$ are arbitrary nonnegative integers and $s_{1}+s_{2}+\cdots+s_{m}=n$.

Observe that the number of successes for each possible outcome $E_{i}$ after $n$ trials can be approximated using (2). ${ }^{8}$ Now we return to BFWCs by stating the following.

Observation 1 Inserting $N$ packets of "Class I traffic" into a BFWC with $m$ counters and $k$ independent hash functions that distribute the packets equiprobably into the $m$ counters, ${ }^{9}$ is equivalent to equiprobably throwing $n=N k$ packets into $m$ bins.

Below we use the terms counter and bin interchangeably. Consider the following experiment.

8 The accuracy of the Poisson approximation to the multinomial distribution is well-studied (e.g., [2, 5, 25]). A Normal distribution may also be used as an approximation to $b(s ; n, p)$ for large $n$ [18].

9 i.e., the probability of throwing a packet into counter $i$ is $1 / m$, for all $1 \leq i \leq m$. 
Table 8 Poisson approximation to $P[k, t, m, N]$ after inserting $N=$ 150,000 packets into a BFWC with $k=4$ hash functions and $m=$ $1,048,576$ counters

\begin{tabular}{ll}
\hline$t$ & $P(k, t, m, N)$ \\
\hline 2 & $1.75 \times 10^{-7}$ \\
3 & $6.50 \times 10^{-11}$ \\
4 & $1.03 \times 10^{-14}$ \\
5 & $8.02 \times 10^{-19}$ \\
6 & $3.43 \times 10^{-23}$ \\
7 & $8.69 \times 10^{-28}$ \\
8 & $1.38 \times 10^{-32}$ \\
9 & $1.45 \times 10^{-37}$ \\
10 & $1.04 \times 10^{-42}$ \\
11 & $5.31 \times 10^{-48}$ \\
12 & $1.98 \times 10^{-53}$ \\
13 & $5.93 \times 10^{-59}$ \\
14 & $2.43 \times 10^{-63}$ \\
15 & $1.52 \times 10^{-64}$ \\
\hline
\end{tabular}

\section{Experiment 1 Throw equiprobably $n$ random packets into $m$ bins.}

We are interested in finding a threshold $t$ such that, after performing Experiment 9, the probability that $k$ random bins have more than $t$ packets is "sufficiently small" to be accepted according to our context (i.e., a tolerable number of false alarms due to "Class I traffic"). Applying (3) may be impractical since $m$ and $n$ may be large. Instead we use equation (2) to approximate the number of packets in each bin.

Example 1 Consider a software implementation of a BFWC with $m=1,048,576$ counters and $k=4$ hash functions. After inserting $N=150,000$ random packets into the BFWC, the mean (expected) number of packets per bin is 0.572205 . We want to set the value of $t$ such that the probability that $k=4$ counters taken at random each hold more than $t$ packets is "sufficiently small". The probability that $k$ random bins all have counters exceeding $t$, denoted $P[k, t, m, N]$, is approximated using (2) as:

$P[k, t, m, N] \approx\left(1-\sum_{i=0}^{t} e^{-n / m} \frac{(n / m)^{i}}{i !}\right)^{k}$.

Table 8 shows this approximation for several values of $t$. Based on the table, a network operator can choose an appropriate value for $t$ according to the number of false alarms tolerable over some time period, under "Class I traffic".

The expected number of false alarms while inserting $N$ packets is $\sum_{i=1}^{N} P[k, t, m, i]$. For large values of $i, P[k, t, m, i]$ can be estimated using (4). For small values of $i, P[k, t, m, i]$ becomes negligibly small. Observe that $P[k, t, m, i]>P\left[k, t, m, i^{\prime}\right]$ for $i>i^{\prime}$. Therefore, a very coarse upper bound for the expected number of false alarms is $\sum_{i=1}^{N} P[k, t, m, i]<N \cdot P[k, t, m, N]$.

\section{References}

1. Anderson, T., Mahajan, R., Spring, N., Wetherall, D.: Rocketfuel: An ISP topology mapping engine (2003). http://www.cs.washington.edu/research/networking/rocketfuel/ [Accessed: August 2, 2003]

2. Barbour, A., Holst, L., Janson, S.: Poisson Approximation. Oxford University Press, New York (1992)

3. Bertsekas, D., Gallager, R.: Data Networks. Prentice Hall, Englewood Cliffs, NJ (1992)
4. Bloom, B.: Space/time trade-offs in hash coding with allowable errors. Commun. ACM 13(17), 422-426 (1970)

5. Boutsikas, M., Koutras, M.: On the number of overflown urns and excess balls in an allocation model with limited urn capacity. Stat. Plan. Inference 104, 259-286 (2002)

6. Broder, A., Kumar, R., Maghoul, F., Raghavan, P., Rajagopalan, S., Stata, R., Tomkins, A., Wiener, J.: Graph structure in the Web. Newblock Comput. Netw. 33(1-6), 309-320 (2000)

7. Broder, A., Mitzenmacher, M.: Network applications of Bloom filters: A survey. Internet Math. 1(4), 485-509 (2003-2004)

8. CERIAS Intrusion Detection Research Group, T.: Digging for worms, fishing for answers. In: Proceedings of the Annual Computer Security Application Conference (ACSAC'02). Las Vegas (2002)

9. Chen, X., Heidemann, J.: Detecting early worm propagation through packet matching. Tech. Rep. ISI-TR-2004-585, University of Southern California (2004)

10. Cormen, T., Leiserson, C., Rivest, R., Stein, C.: Introduction to Algorithms, 2nd edn. MIT Press, McGraw-Hill, New York (2001)

11. Crosby, S., Wallach, D.: Denial of service via algorithmic complexity attacks. In: Proceedings of the 12th USENIX Security Symposium. Washington, DC (2003)

12. Daley, D., Gani, J.: Epidemic Modelling: An Introduction. Cambridge University Press, Cambridge, UK (1999)

13. Dharmapurikar, S., Krishnamurthy, P., Sproull, T., Lockwood, J.: Deep packet inspection using parallel Bloom filters. In: Symposium on High Performance Interconnects (HotI), pp. 44-51. Stanford, CA (2003)

14. Dharmapurikar, S., Krishnamurthy, P., Taylor, D.: Longest prefix matching using Bloom filters. In: Proceedings of the Special Interest Group on Data Communication (SIGCOMM'03), pp. 201212. Karlsruhe, Germany (2003)

15. Dharmapurikar, S., Paxson, V.: Robust TCP stream reassembly in the presence of adversaries. In: Proceedings of the 14th USENIX Security Symposium. Baltimore (2005)

16. Faloutsos, M., Faloutsos, P., Faloutsos, C.: On power-law relationships of the Internet topology. In: Proceedings of the Special Interest Group on Data Communication (SIGCOMM'99), pp. 251262. Boston/Cambridge, MA (1999)

17. Fan, L., Cao, P., Almeida, J., Broder, A.: Summary cache: A scalable wide-area Web cache sharing protocol. IEEE/ACM Trans. Netw. 8(3), 281-293 (2000)

18. Feller, W.: An Introduction to Probability Theory and its Applications, vol. 1, 3rd edn. Wiley, New York (1968)

19. Fyodor: The art of port scanning. Phrack Mag. 7(51) (1997). URL: http://www.phrack.org [Accessed: March 6, 2003]

20. Garey, M., Johnson, D.: Computers and Intractability: A Guide to the Theory of NP-Completeness. W. H. Freeman, New York (1979)

21. Goh, E.J.: Secure indexes. Cryptology ePrint Archive, Report 2003/216 (2003). URL: http://eprint.iacr.org/2003/216/ [Accessed: January 7, 2004]

22. Grembowski, T., Lien, R., Gaj, K., Nguyen, N., Bellows, P., Flidr, J., Lehman, T., Schott, B.: Comparative analysis of the hardware implementations of hash functions SHA-1 and SHA-512. In: Proceedings of Information Security Conference (ISC 2002), Lecture Notes in Computer Science, vol. 2433, pp. 75-89. Springer, Sao Paulo, Brazil (2002)

23. Handley, M., Kreibich, C., Paxson, V.: Network intrusion detection: Evasion, traffic normalization, and end-to-end protocol semantics. In: Proceedings of the 10th USENIX Security Symposium. Washington, DC (2001)

24. Horne, B., Matheson, L., Sheehan, C., Tarjan, R.: Dynamic selfchecking techniques for improved tamper resistance. In: Proceedings of the First ACM Workshop on Digital Rights Management (DRM 2001), Lecture Notes in Computer Science, vol. 2320, pp. 141-159. Springer, Berlin Heidelberg New York (2002)

25. Joag-Dev, K., Proschan, F.: Negative association of random variables, with applications. Ann. Stat. 11(1), 286-295 (1983) 
26. Jung, J., Paxson, V., Berger, A., Balakrishnan, H.: Fast portscan detection using sequential hypothesis testing. In: Proceedings of the 2004 IEEE Symposium on Security and Privacy. Oakland (2004)

27. Kim, H.A., Karp, B.: Autograph: Toward automated, distributed worm signature detection. In: Proceedings of 13th USENIX Security Symposium. San Diego, CA (2004)

28. Kumar, A., Xu, J., Li, L., Wang, J.: Space-code Bloom filter for efficient traffic flow measurement. In: Proceedings of IMC. Miami Beach, FL (2003)

29. Levy, E.: Worm propagation and generic attacks. IEEE Secur. Priv. 3(2), 63-65 (2005)

30. Liljenstam, M.: Modeling of security and systems. A network worm modeling package for SSFNet (2003). http://www.crhc. uiuc.edu/mili/research/ssf/worm/ [Accessed: September 10, 2004]

31. Mahajan, R., Spring, N., Wetherall, D., Anderson, T.: Inferring link weight using end-to-end measurements. In: Proceedings of the Internet Measurement Workshop 2002 (IMW'02). Marseille, France (2002)

32. Matrawy, A., van Oorschot, P., Somayaji, A.: Mitigating network denial-of-service through diversity-based traffic management. In: Proceedings of the 3rd Annual Conference on Applied Cryptography and Network Security (ACNS 2005), Lecture Notes in Computer Science, vol. 3531, pp. 104-121. Springer, New York (2005)

33. McHugh, J.: Testing intrusion detection systems: A critique of the 1998 and 1999 DARPA intrusion detection system evaluations as performed by Lincoln Laboratory. ACM Trans. Inf. Syst. Secur. (TISSEC) 3(4), 262-294 (2000)

34. MIT Lincoln Laboratory: DARPA intrusion detection evaluation: Data sets (1999). http://www.ll.mit.edu/IST/ideval/data/ data_index.html [Accessed: April 1, 2004]

35. Mitzenmacher, M.: Compressed Bloom filters. In: Proceedings of the 20th Annual ACM Symposium on Principles of Distributed Computing (PODC 2001), pp. 144-150. Newport, RI (2001)

36. Moore, D., Paxon, V., Savage, S., Shannon, C., Staniford, S., Weaver, N.: Inside the Slammer worm. IEEE Secur. Priv. 1(4), 33-39 (2003)

37. Nachenberg, C.: Computer virus-antivirus coevolution. Commun. ACM 40(1), 46-51 (1997)

38. Nevelsteen, W., Preneel, B.: Software performance of universal hash functions. In: Proceedings of Eurocrypt'99, pp. 24-41. Prague, Czech Republic (1999)

39. Newsome, J., Karp, B., Song, D.: Polygraph: Automatically generating signatures for polymorphic worms. In: Proceedings of the 2005 IEEE Symposium on Security and Privacy. Oakland, CA (2005)

40. NS-2: The network simulator - NS-2 (2003). http://www.isi.edu/ nsnam/ns/ [Accessed: September 10, 2003]

41. Onut, I.V., Zhu, B., Ghorbani, A.: A novel visualization technique for network anomaly detection. In: Proceedings of the 2nd Annual Conference on Privacy, Security and Trust. Fredericton, Canada (2004)

42. OPNET Technologies Inc.: Opnet modeler (2003). http://www. opnet.com [Accessed: September 10, 2003]

43. Park, K., Lee, H.: On the effectiveness of route-based packet filtering for distributed DoS attack prevention in power-law internets. In: Proceedings of the Special Interest Group on Data Communication (SIGCOMM'01). San Diego, CA (2001)

44. Ptacek, T.H., Newsham, T.N.: Insertion, evasion and denial of service: Eluding network intrusion detection. Tech. rep., Secure Networks, Inc. (1998). http://www.aciri.org/vern/Ptacek-NewshamEvasion-98.ps [Accessed: November 6, 2005]

45. Rabin, M.: Fingerprinting by random polynomials. Technical Report TR-15-81, Center for Research in Computing Technology, Harvard University, Cambridge, MA (1981)
46. Shanmugasundaram, K., Brönnimann, H., Memon, N.: Payload attribution via hierarchical Bloom filters. In: Proceedings of the 11th ACM Conference on Computer and Communications Security (CCS'04). Washington, DC (2004)

47. Shannon, C., Moore, D.: The spread of the Witty worm (2004). http://www.caida.org/analysis/security/witty/ [Accessed: June 18, 2004]

48. Singh, S., Estan, C., Varghese, G., Savage, S.: The EarlyBird system for real-time detection of unknown worms. Technical Report CS2003-0761, University of California, San Diego, CA (2003)

49. Singh, S., Estan, C., Varghese, G., Savage, S.: Automated worm fingerprinting. In: Proceedings of the 6th USENIX Symposium on Operating Systems Design \& Implementation (OSDI'04). San Francisco (2004)

50. Snoeren, A., Partridge, C., Sanchez, L., Jones, C., Tchakountio, F., Kent, S., Strayer, W.: Hash-based IP traceback. In: Proceedings of the Special Interest Group on Data Communication (SIGCOMM'01). San Diego, CA (2001)

51. Spring, N., Mahajan, R., Wetherall, D.: Measuring ISP topologies with Rocketfuel. In: Proceedings of the Special Interest Group on Data Communication (SIGCOMM'02). Pittsburgh, PA (2002)

52. SSFNet: Scalable simulation framework network models (2003). http://www.ssfnet.org/homePage.html [Accessed: September 10, 2003]

53. Toth, T., Kruegel, C.: Connection-history based anomaly detection. In: Proceedings of the 2002 IEEE Workshop on Information Assurance and Security. New York (2002)

54. Twycross, J., Williamson, M.: Implementing and testing a virus throttle. In: Proceedings of the 12th USENIX Security Symposium. Washington, DC (2003)

55. Valdes, A., Fong, M.: Scalable visualization of propagating Internet phenomena. In: Proceedings of the ACM Workshop on Visualization and Data Mining for Computer Security. Washington, DC (2004)

56. Vargas Martin, M.: A monitoring system for mitigating fast propagating worms in the network infrastructure. In: Proceedings of the 18th IEEE Canadian Conference on Electrical and Computing Engineering (CCECE'05). Saskatoon, Canada (2005)

57. Venkataraman, S., Song, D., Gibbons, P., Blum, A.: New streaming algorithms for fast detection of superspreaders. In: The Internet Society Proceedings of the Network and Distributed System Security Symposium (NDSS'05). San Diego, CA (2005)

58. Wang, K., Stolfo, S.: Anomalous payload-based network intrusion detection. In: Proceedings of the Seventh International Symposium on Recent Advances in Intrusion Detection (RAID 2004). Sophia Antipolis, France (2004)

59. Watts, D.: Small Worlds: The Dynamics of Networks Between Order and Randomness. Princeton University Press, Princeton, NJ (1999)

60. Weaver, N., Paxson, V., Staniford, S., Cunningham, R.: A taxonomy of computer worms. In: Proceedings of ACM WORM'03. Washington, DC (2003)

61. Weaver, N., Staniford, S., Paxson, V.: Very fast containment of scanning worms. In: Proceedings of the 13th USENIX Security Symposium. San Diego, CA (2004)

62. Williamson, M.: Throttling viruses: Restricting propagation to defeat malicious mobile code. In: Proceedings of the Annual Computer Security Application Conference (ACSAC'02). Las Vegas (2002)

63. Zou, C., Gong, W., Towsley, D.: Code Red worm propagation modeling and analysis. In: Proceedings of the 9th ACM Conference on Computer and Communications Security (CCS'02). Washington, DC (2002) 


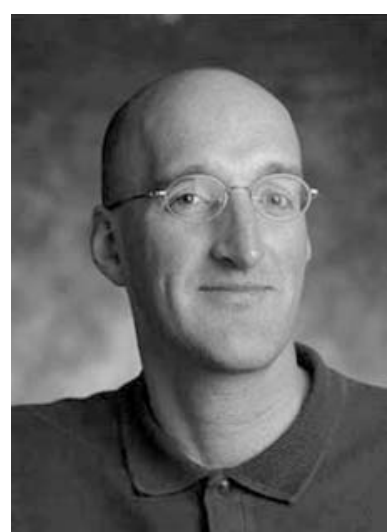

P. van Oorschot (Ph.D. Waterloo, 1988) is a Professor in the School of Computer Science at Carleton University, and Canada Research Chair in Network and Software Security. $\mathrm{He}$ is the founding director of Carleton's Digital Security Group. He has worked in research and development in cryptography and network security, including at BellNorthern Research (Ottawa), and Entrust Technologies (Ottawa) as VP and Chief Scientist. He is coauthor of the standard reference Handbook of Applied Cryptography. His current research interests include authentication and identity management, network security, software protection, and security infrastructures.

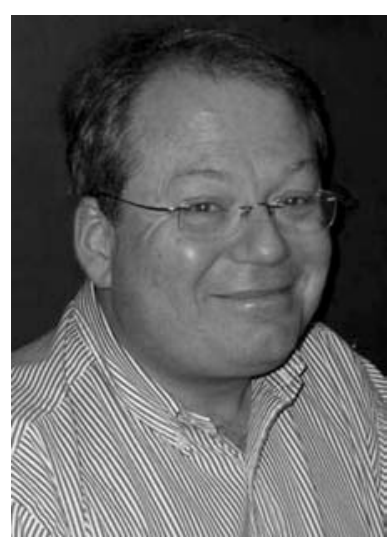

J.-M. Robert is a Principal Security Researcher at Alcatel in Ottawa, Ontario. His research interests are network and telecom infrastructure security, focusing mainly on denial-of-service attacks and worm propagation. Previously, Dr. Robert worked as Security Director for the North American Development Center of Gemplus International as well as Professor at the Université du Québec à Chicoutimi. Dr. Robert received a Ph.D. in Computer Science from McGill University.

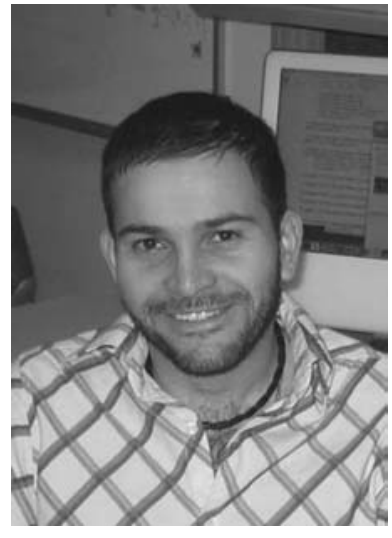

M. Vargas Martin is an Assistant Professor at the University of Ontario Institute of Technology (Oshawa, Canada), with faculty appointments in Business and Information Technology, as well as Engineering and Applied Science. He was previously a post-doctoral researcher at Carleton University supported in part by Alcatel Canada. He holds a Ph.D. in Computer Science (Carleton University, 2002), a Masters degree in Electrical Engineering (Cinvestav, Mexico, 1998), and a Bachelor of Computer Science (Universidad Autónoma de Aguascalientes, Mexico, 1996). His current research interests include network and host-based intrusion detection and reaction, mitigation of denial-of-service (DoS) and distributed DoS attacks, Web modeling and optimization, Internet connectivity, and interconnection protocols. 